\title{
Shielding calculation for the Proton-Therapy-Center in Prague, Czech Republic
}

\author{
T. URBAN* ${ }^{*}$ J. KLUSON
}

(Manuscript received 19 March 2012, accepted 2 July 2012)

\begin{abstract}
The study of radiation fields around the cyclotron room was carried out for the Proton-Therapy-Center in Prague (Czech Republic). The $230 \mathrm{MeV}$ proton cyclotron will be installed there as well as additional components located along the beampath. The ambient doses were computed by taking into account 3 major sources of secondary radiation (neutron and photon) - the cyclotron, energy degrader and its collimator. The fluxes of neutrons/photons produced by protons were computed using Monte Carlo code MCNPX ${ }^{\mathrm{TM}}$ 2.5.0. Energy and angular distributions were computed for various targets and proton beam energies. Different physical models of high-energy proton interactions were studied as well. The annual charge at the cyclotron exit was based on the "patient model" (distribution of treatment type) established for a similar therapy center in Essen (Germany). Calculated data were used as an approximation of the radiation field of the most significant sources of radiation (graphite degrader, tantalum collimator). Fluence-to-dose conversion factors from ICRP-74 were used for the annual dose equivalent evaluation. With these results, the shielding analysis in the Therapy-Center was performed in the context of the current legislation requirements.
\end{abstract}

Keywords: Monte Carlo / proton / shielding / therapy

\section{Introduction}

Proton therapy is becoming an increasingly popular modality in radiation therapy, most often for the treatment of malignant and non-malignant tumors. A known advantage of proton therapy is its ability to deliver a radiation dose with high precision to the tumor while the surrounding healthy tissue and organs are irradiated minimally. Different types of tumors can be treated by using proton therapy; for instance, tumors of the brain and base of the skull, head and neck tumors, malignant melanoma of the eye, lung tumors, prostate cancer, tumors and metastases of the spine, tumors of the abdomen and pelvis, etc. In the context of these wide spectra of proton therapy application and different depths of target volumes it is necessary

Czech Technical University in Prague, Faculty of Nuclear Sciences and Physical Engineering, Brehova 7, 11519 Prague 1, Czech Republic.

*e-mail: tomas.urban@fjfi.cvut.cz 
to use different energies for treatment and so it is necessary to slow down accelerated protons to energies suitable for the specific depth of the target volume.

The cost of the building can be over 100 millions EUR and the shielding can have a significant impact on the cost; on the other hand, it must be designed to adequately protect personnel and the public. The primary purpose of this study is to design a shield that will be in agreement with the current legislation requirements. The design of the Prague Proton-Therapy-Center is similar to the existing facility in Essen, Germany. Thus, some calculations in this article are also an independent assessment of proven shielding design.

In this work, the stochastic approach was used for verification of the shielding design. The fluxes of secondary radiation (neutrons and photons) produced by protons were computed using Monte Carlo code $\mathrm{MCNPX}^{\mathrm{TM}}$, version 2.5.0. The ambient dose equivalents at selected critical points around the shielding structure were calculated using fluence-to-dose conversion factors from the publication 74 of the International Commission on Radiological Protection (ICRP, 1996). The influence of the high-energy nuclear interaction model choice was tested as well.

\section{Materials and methods}

\subsection{Shielding design aims}

The primary design aims are to limit the annual dose equivalent behind the shielding walls to $<1 \mathrm{mSv}$ (outside of the proton therapy facility) and $<20 \mathrm{mSv}$ (inside of the proton therapy facility, i.e. in control rooms, in corridors, etc.), respectively. Dose equivalents were estimated assuming a 16-hour daily shift, 6 working days per week, all year round (Stichelbaut, 2008). All calculations were also based on technology contractor information as well as a patient model and distribution of treatment type according to the intended operation of the Proton-Therapy-Center (Stichelbaut, 2007; Stichelbaut, 2008).

\subsection{Main sources of radiation and spectra of secondary radiation around them}

This work aimed to verify shielding barriers around the room with acceleratorcyclotron-accelerating $230 \mathrm{MeV}$ protons. Thus, the main sources in the cyclotron room are cyclotron and energy selection systems. The energy selection system is composed of two main components - a degrader and its collimator. The degrader is made of graphite (density $1.8 \mathrm{~g} / \mathrm{cm}^{3}$ ) and is designed to slow down the proton 
SHIELDING CALCULATION FOR THE PROTON-THERAPY-CENTER IN PRAGUE

TABLE I

Parameters of the cylinder target used in simulation instead of graphite degrader.

\begin{tabular}{llcrl}
\hline $\begin{array}{l}\text { Energy of protons at the end of the } \\
\text { target/cylinder }\end{array}$ & $\mathbf{1 9 0} \mathbf{M e V}$ & $\mathbf{1 5 0} \mathbf{~ M e V}$ & $\mathbf{1 1 0 ~ M e V}$ & $\mathbf{7 0 ~ M e V}$ \\
\hline cylinder radius $[\mathrm{cm}]$ & & \multicolumn{2}{c}{$22.385 \mathrm{~cm}$} \\
\hline cylinder length $[\mathrm{cm}]$ & $5.686 \mathrm{~cm}$ & $10.634 \mathrm{~cm}$ & $14.739 \mathrm{~cm}$ & $17.865 \mathrm{~cm}$ \\
\hline
\end{tabular}

TABLE II

Parameters of the cylinder target used in simulation instead of tantalum collimator.

\begin{tabular}{lccccc}
\hline $\begin{array}{l}\text { Energy of protons entering } \\
\text { the target/cylinder }\end{array}$ & $\mathbf{7 0} \mathbf{M e V}$ & $\mathbf{1 1 0} \mathbf{M e V}$ & $\mathbf{1 5 0} \mathbf{M e V}$ & $\mathbf{1 9 0} \mathbf{M e V}$ & $\mathbf{2 3 0} \mathbf{M e V}$ \\
\hline cylinder radius [cm] & $0.5599 \mathrm{~cm}$ & $1.2122 \mathrm{~cm}$ & $2.0515 \mathrm{~cm}$ & $3.0503 \mathrm{~cm}$ & $4.1877 \mathrm{~cm}$ \\
cylinder length [cm] & $0.5599 \mathrm{~cm}$ & $1.2122 \mathrm{~cm}$ & $2.0515 \mathrm{~cm}$ & $3.0503 \mathrm{~cm}$ & $4.1877 \mathrm{~cm}$ \\
\hline
\end{tabular}

energy to energies that are indicated for the treatment (in this study 5 energy categories were taken into account: $230 \mathrm{MeV}, 190 \mathrm{MeV}, 150 \mathrm{MeV}, 110 \mathrm{MeV}$ and $70 \mathrm{MeV}$ ). The collimator is made of tantalum (density $16.6 \mathrm{~g} / \mathrm{cm}^{3}$ ) and is used for narrowing the scattered proton beam after the degrader (Stichelbaut, 2007, 2008).

The cyclotron. The cyclotron extraction efficiency is assumed to be $\sim 50 \%$, i.e. $~ 50 \%$ of the beam is lost in the cyclotron (Stichelbaut, 2007). All beam losses in the cyclotron were assumed at the extraction radius, i.e. at the full energy, $230 \mathrm{MeV}$, where the proton beam interacts with a copper material. The thickness of a copper layer (in all directions - like a "hollow" cylinder with the source on the inner surface) in simulations is bigger than the range of $230 \mathrm{MeV}$ protons in a given material/copper. The cyclotron itself is shielded in all directions by at least $40 \mathrm{~cm}$ of iron (density $7.86 \mathrm{~g} / \mathrm{cm}^{3}$ ), i.e. an iron layer (the shape of the "hollow" cylinder around the copper one, see above) of $40 \mathrm{~cm}$ thickness was considered in simulations as well (Stichelbaut, 2008).

The graphite degrader. Secondary radiation spectra were calculated for the narrow $230 \mathrm{MeV}$ proton beam incident perpendicular to the middle of the base of the graphite cylinder. The dimensions of these targets/cylinders are shown in Table I. In all cases the radius of cylinder is greater than the range of $230 \mathrm{MeV}$ protons and the length of the cylinder is designed so that the proton beam energy at the exit of the cylinder is equal to the desired energy (used for treatment).

The collimator. The dimensions of the target/cylinder substituting for purposes of collimator simulations are shown in Table II. In this case the radius and length of the target/cylinder are equal to each other and both dimensions are greater than the range of protons incident on a tantalum target/cylinder. Also, in 


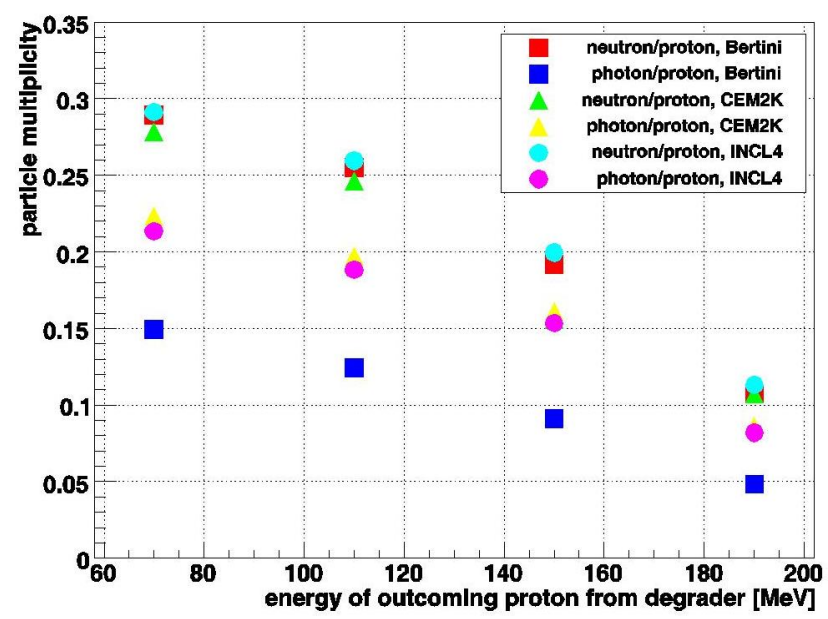

Figure 1-(Color online) Multiplicities of particles emitted from cylindrical target made of graphite (degrader).

the case of the collimator, the proton beam was considered as narrow and as perpendicularly incident on a base of the target/cylinder.

In simulations, the geometric center of a cylinder/target was placed at the origin of the coordinate system. A cylinder axis coincides with the $x$-axis as well as the proton beam incident on the target/cylinder in the middle of the cylinder base. The cylinders were surrounded by air. At the sphere of radius of 1 meter secondary particles emitted from the target were registered. Neutrons and photons were identified as the main components of the secondary radiation. In Figures 1 and 2 multiplicities of particles emitted from targets are shown. Examples of some energy and angular spectra of neutrons from various sources of radiation are shown in Figures 3-5. Generally, neutron spectra cover the entire energy interval from zero energy up to the energy of protons impinging on a target. As can be seen in Figures 6 and 7, the energy spectra of photons are not so wide - spectra finished, maximally, at several tens of MeV. Also, the multiplicity of photons is lower than that of neutrons. In all simulations there were three different interaction models of high-energy particles included in $\mathrm{MCNPX}^{\mathrm{TM}}$, version 2.5.0 - Bertini, CEM2K and INCL4. In all cases the intranuclear cascade (INC) interaction model was followed by the evaporation-fission model RAL (Pelowitz, 2005).

In all other (not shown in this paper) cases (different energy of incident protons) the energy and angular spectra had a similar character, for both neutrons and photons. The energy and angular spectra suggest that selection of an appropriate 


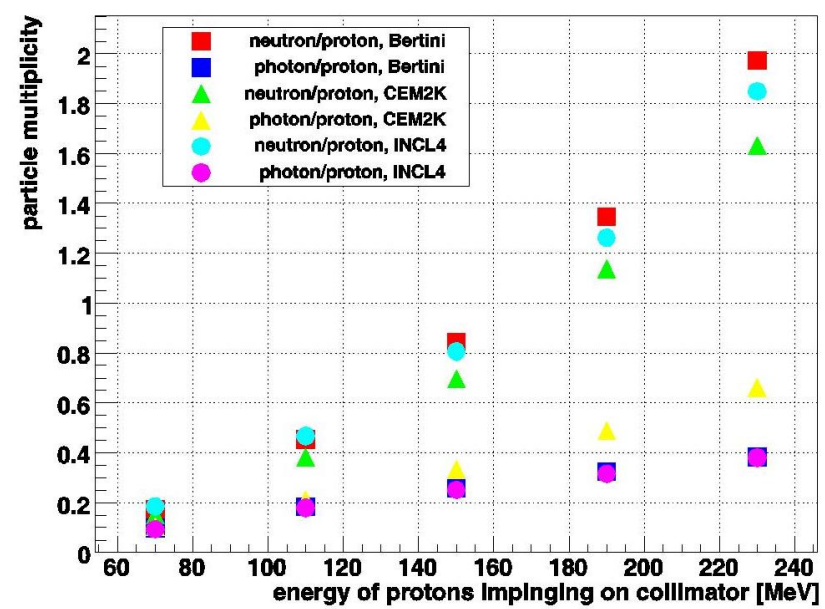

Figure 2-(Color online) Multiplicities of particles emitted from cylindrical target made of tantalum (collimator).

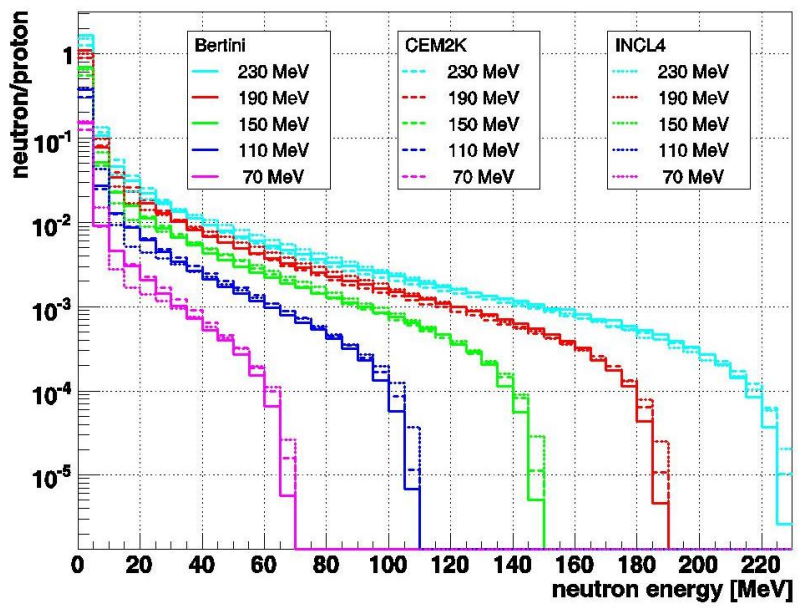

Figure 3-(Color online) Examples of energy spectra of neutrons emitted from tantalum target (collimator) for various nuclear interaction calculation models. 


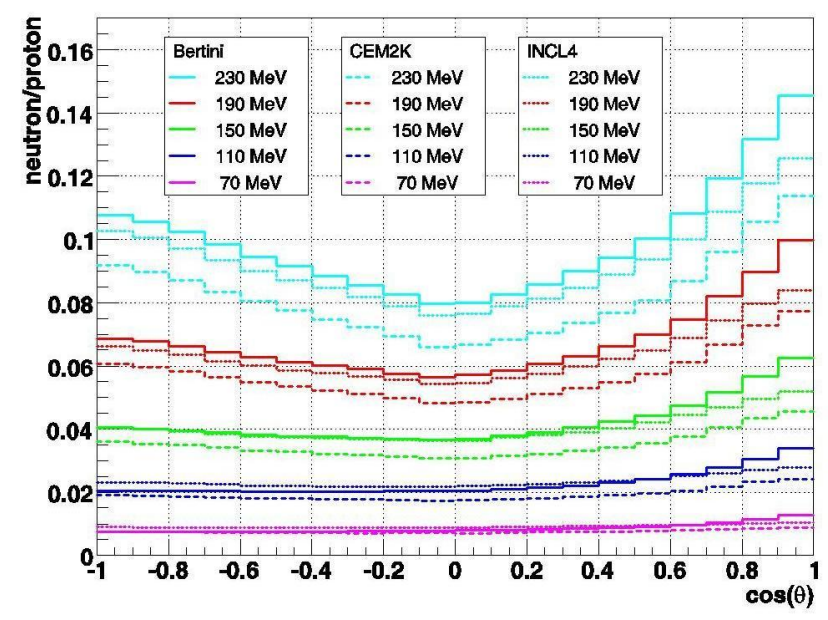

Figure 4-(Color online) Examples of angular spectra of neutrons emitted from tantalum target (collimator) for various nuclear interaction calculation models.

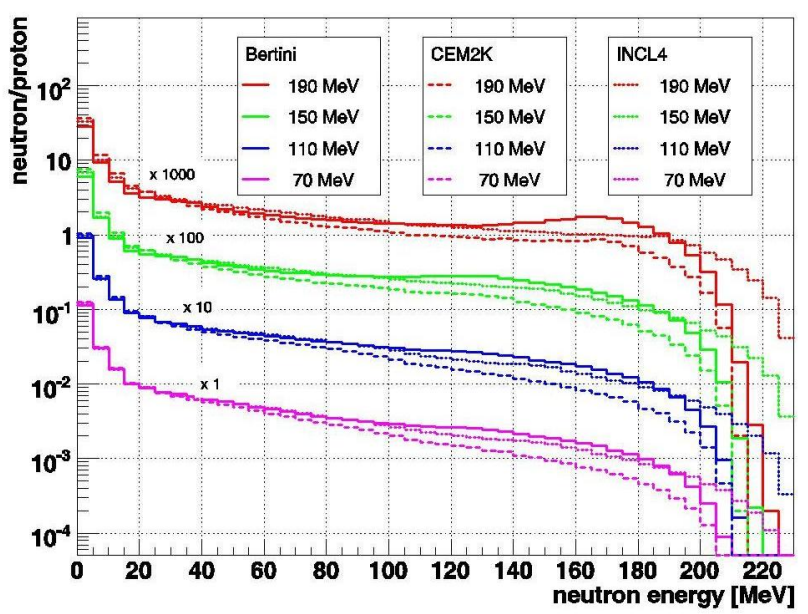

Figure 5 -(Color online) Examples of energy spectra of neutrons emitted from graphite target (degrader) for various nuclear interaction calculation models. 


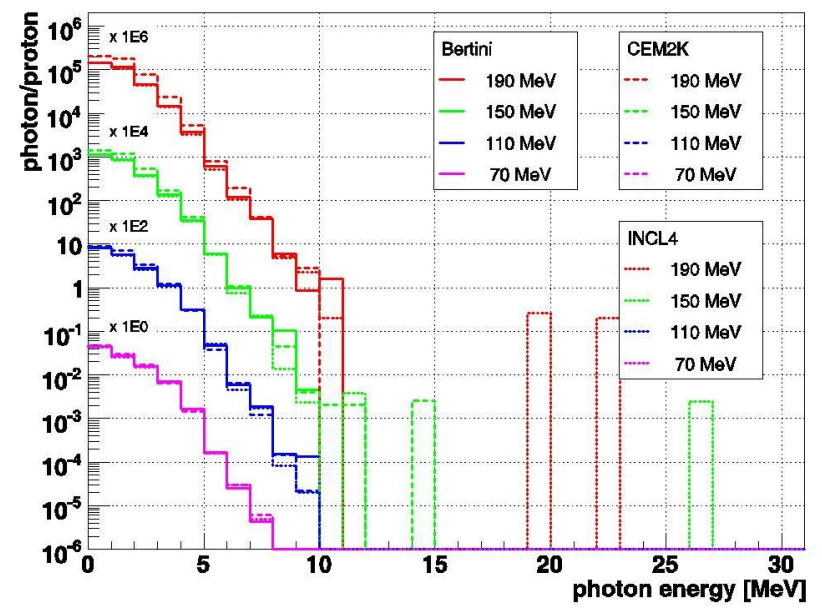

Figure 6-(Color online) Examples of energy spectra of photons emitted from tantalum target (collimator) for various nuclear interaction calculation models.

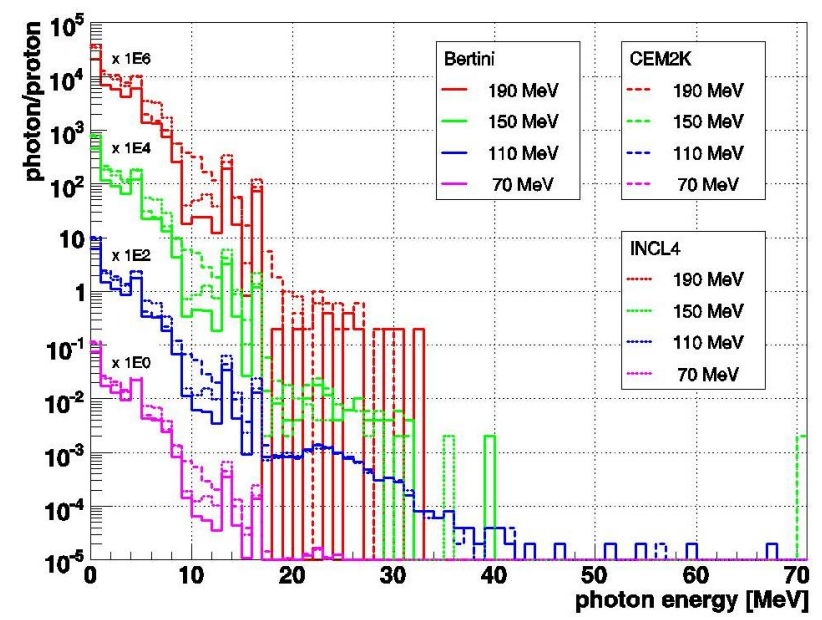

Figure 7 -(Color online) Examples of energy spectra of photons emitted from graphite target (degrader) for various nuclear interaction calculation models. 
TABLE III

Annual charge at cyclotron exit for selected energies.

\begin{tabular}{lccc}
\hline Energy & $\begin{array}{c}\text { Beam current } \\
\text { @ cyclotron exit } \\
\text { [nA] }\end{array}$ & $\begin{array}{c}\text { Irradiation time } \\
\text { [hours/year] }\end{array}$ & $\begin{array}{c}\text { Annual charge } \\
\text { @ cyclotron exit } \\
\text { [nA.h/year] }\end{array}$ \\
\hline $\mathbf{7 0 ~ M e V + 1 1 0 ~ M e V ~}$ & 300 & 7 & 2100 \\
$\mathbf{1 5 0} \mathbf{~ M e V}$ & 147 & 300 & 44100 \\
$\mathbf{1 9 0} \mathbf{M e V}$ & 50 & 600 & 30000 \\
$\mathbf{2 3 0} \mathbf{M e V}$ & 16 & 375 & 6000 \\
\hline
\end{tabular}

model for the shielding calculations/verifications is not so easy with these results. Use of any nuclear interaction model has its pros and cons. One leads to a greater multiplicity of secondary particles in the entire energy spectrum, while another only at high energies (hundreds of $\mathrm{MeV}$ ). Thus, all the mentioned nuclear interaction models were tested and compared for shielding calculations/verifications as well.

\subsection{Shielding calculations}

All three INC models of interaction of high-energy particles were used in the $\mathrm{MCNPX}^{\mathrm{TM}}$ - Bertini, CEM2K and INCL4. In all three cases the INC models were followed by the evaporation-fission model RAL. The impact of the evaporation model choice is not so crucial for shielding calculation design and so the default values were left.

All the models used give comparable/similar results with no major deviations from the relevant radiation protection with an impact on legislative requirement compliance - see Figure 8. Example cross-sections are located in a vertical plane perpendicular to the walls. In the calculations the only neutron component of the secondary radiation was included. The photon component gives only approximately 1 percent of the contribution to the ambient dose equivalent $H^{*}(10)$. For evaluation of the dose equivalent fluence-to-dose conversion factors for neutrons from publication 74 of ICRP (1996) were used. For the results the expected annual capacity of the Proton Therapy Center due to the number and types of therapeutic interventions were also taken into account (Stichelbaut, 2007, 2008). The estimation of capacity is based on the "patient model" established for a similar therapy center in Essen (Germany). The annual charge of the proton beam at the cyclotron exit is in Table III (Stichelbaut, 2007). Energies $70 \mathrm{MeV}$ and $110 \mathrm{MeV}$, respectively, are in further calculations considered in the ratio 1:1.

Comparison of the results of different models (not just presented in Fig. 8) gives the result that the most conservative choice for the purposes of the shielding analysis design is the Bertini INC model (followed by the RAL evaporation model). The Bertini model is also the fastest one, approximately twice as fast as 

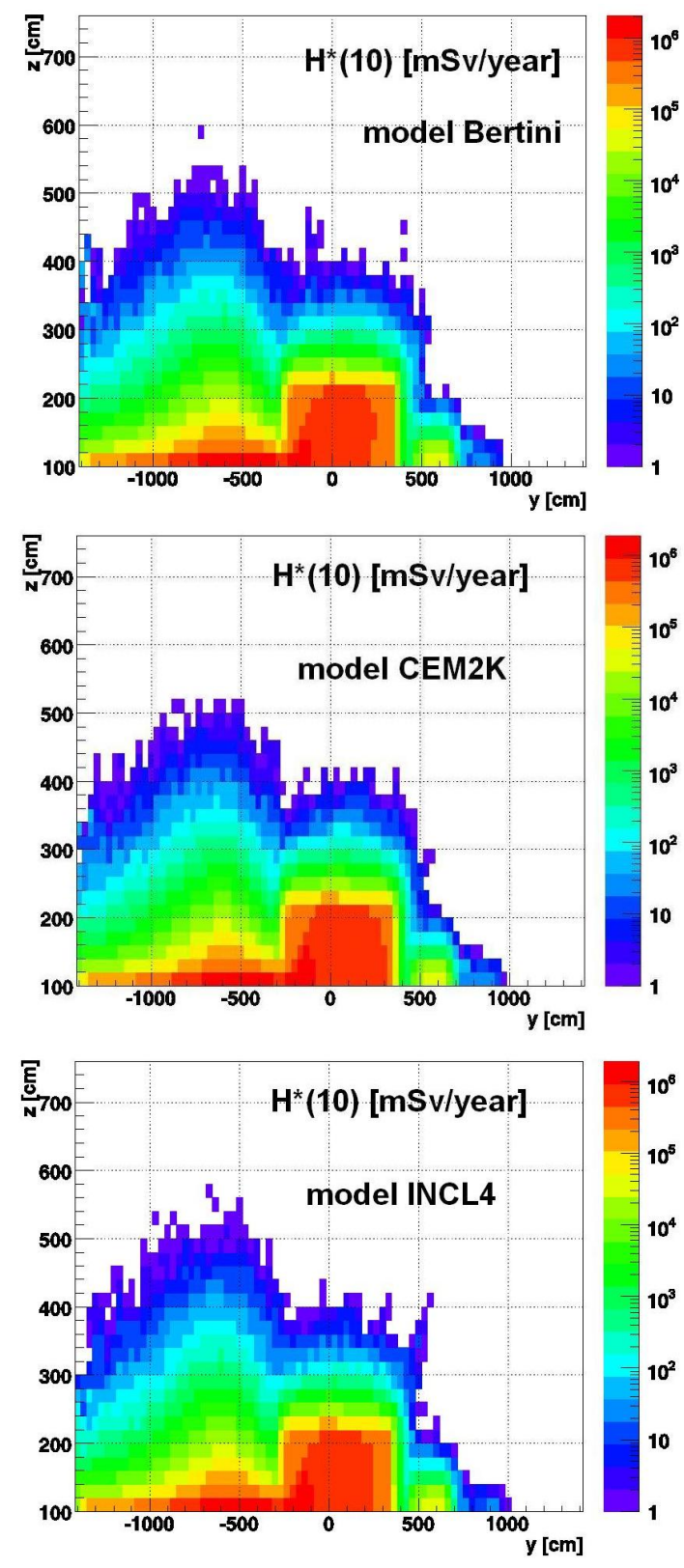

Figure 8-(Color online) Comparison of $2 D$ distribution $H^{*}(10)$ [mSv/year] for different interaction models (top: Bertini, center: CEM2K, bottom: INCL4). 


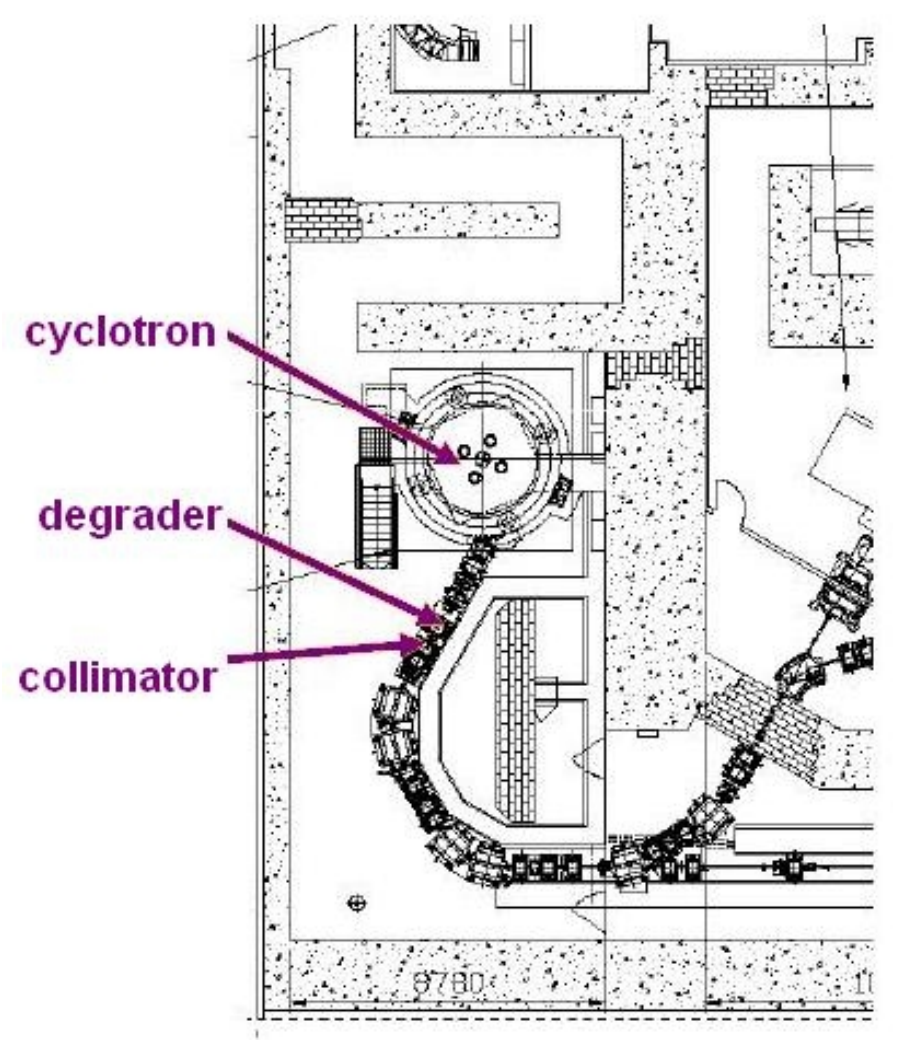

Figure 9 - (Color online) Ground plan of the cyclotron room.

the CEM2K and four times as fast as the INCL4, and so the Bertini INC interaction model was used in the shielding analysis.

Three main sources of radiation were taken into account for the shielding verification - the cyclotron, degrader and its collimator - see Figure 9. These components and their simplification for simulation are described above, in Section 2.2. Accelerator efficiency is considered to be 50 percent. A conservative approach where beam losses are uniformly distributed around the cyclotron at the extraction radius was used. Measured transmission factors for the degrader and collimator were used for calculation. By convention, it was considered that there are no beam losses in the degrader (it means transmission of the degrader is equal to 100 percent) and all beam losses of the degrader and collimator were attributed to collimator transmission. This transmission depends on the energy of the proton beam after the degrader as well as on the beam delivery mode during treatment. 


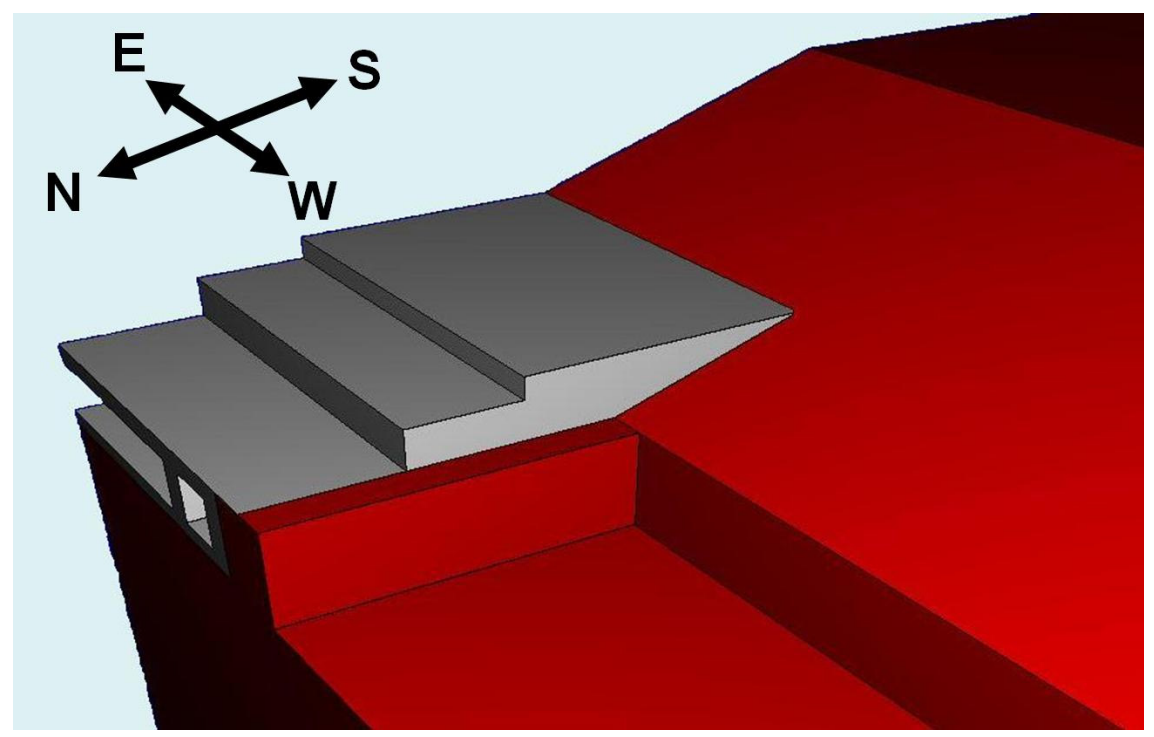

Figure 10 - (Color online) $3 D$ model of the room with a cyclotron and its accessories (gray =concrete, red $=$ soil $)$.

In the Proton Therapy Center both active scanning and passive scattering will be used for treatments. According to the information from the contractor, use of collimator transmission factors for active scanning is a more conservative approach (Stichelbaut, 2007, 2008).

Atomic composition, and even more density, of shielding materials (especially concrete and soil) are essential for neutron (and photon) attenuation. The same material composition of concrete was used as in (Stichelbaut, 2008), where the composition of concrete is based on the NIST database and the composition of soil on average values represented by individual elements in the soil on the site. In comparison with (Stichelbaut, 2008), in this work a reduction of the mass density of the materials used is applied from the real value $2.5 \mathrm{~kg} / \mathrm{m}^{3}$ to $2.3 \mathrm{~kg} / \mathrm{m}^{3}$ (concrete) and from $1.8 \mathrm{~kg} / \mathrm{m}^{3}$ to $1.4 \mathrm{~kg} / \mathrm{m}^{3}$ (soil), respectively. The impact of material composition and density in a real situation in comparison with the values used in the simulations is discussed later (Discussion-section).

As the cyclotron room is located underground (see Fig. 10), the outside concrete walls of the cyclotron room (west and south walls) have reduced thickness followed by soil. The annual doses were also computed inside the fixed beam room (towards the East), at the maze exit (towards the North), inside the main control room (North, next to the maze), and above the cyclotron room roof. 


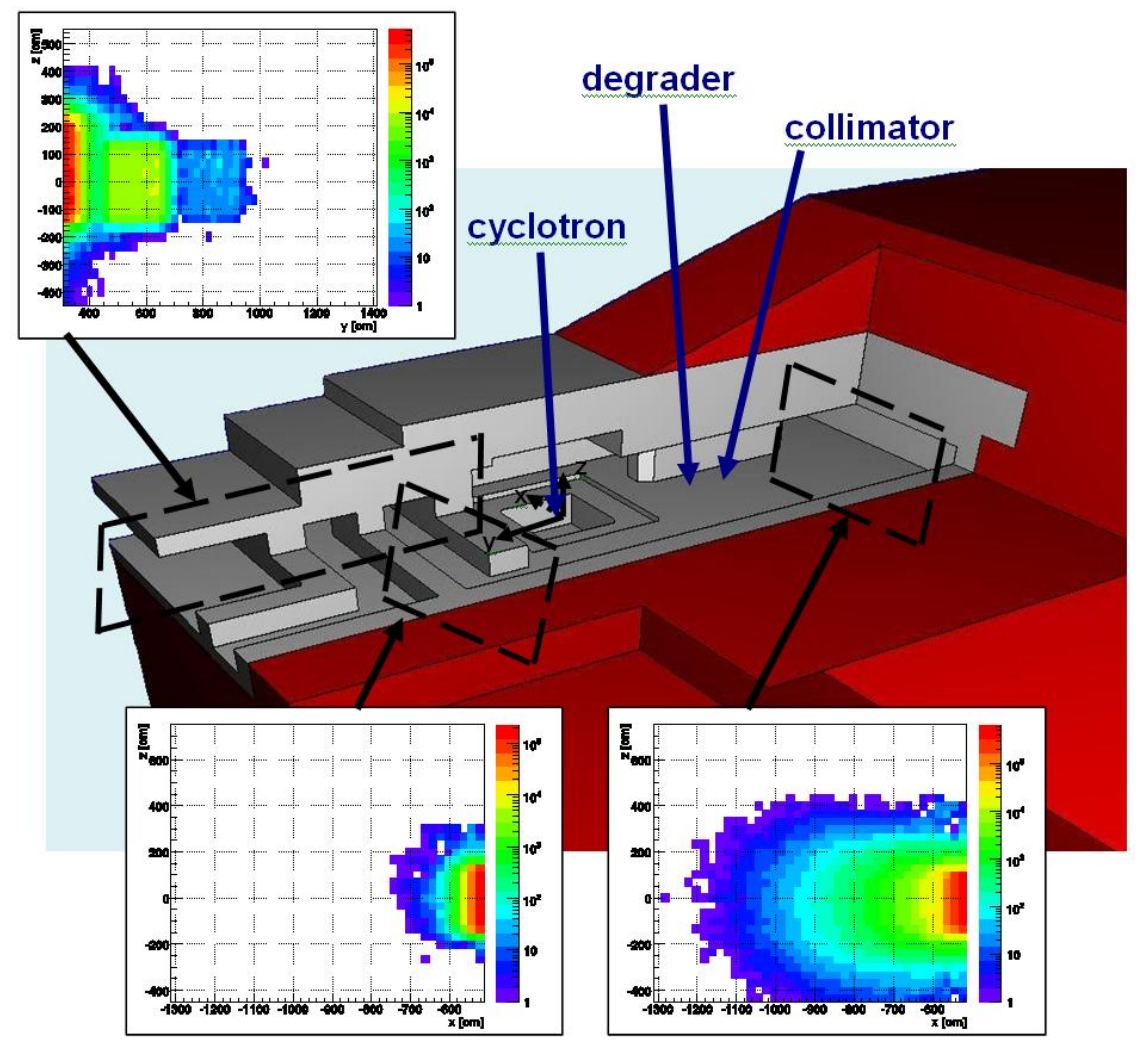

Figure $11-($ Color online $) H^{*}(10)[\mathrm{mSv} /$ year] in selected areas of the west wall and maze of the cyclotron room.

Calculated 3D dose distributions were used to verify the radiation shielding design and the radiation protection optimization.

Figures 11 and 12 show the 2D distributions of the calculated values of the ambient dose equivalent $H^{*}(10)$ [mSv/year] around the room (see 3D drawing of the Proton-Therapy-Center in Prague) with the cyclotron, etc. The presented values of the ambient dose equivalent $H^{*}(10)$ are evaluated in the same way as the values in Figure 8, e.g. using fluence-to-dose conversion factors from publication 74 of ICRP (1996) and the expected annual capacity of the Proton-TherapyCenter. Cross-sections are located in a vertical plane perpendicular to the walls as in Figure 8. 


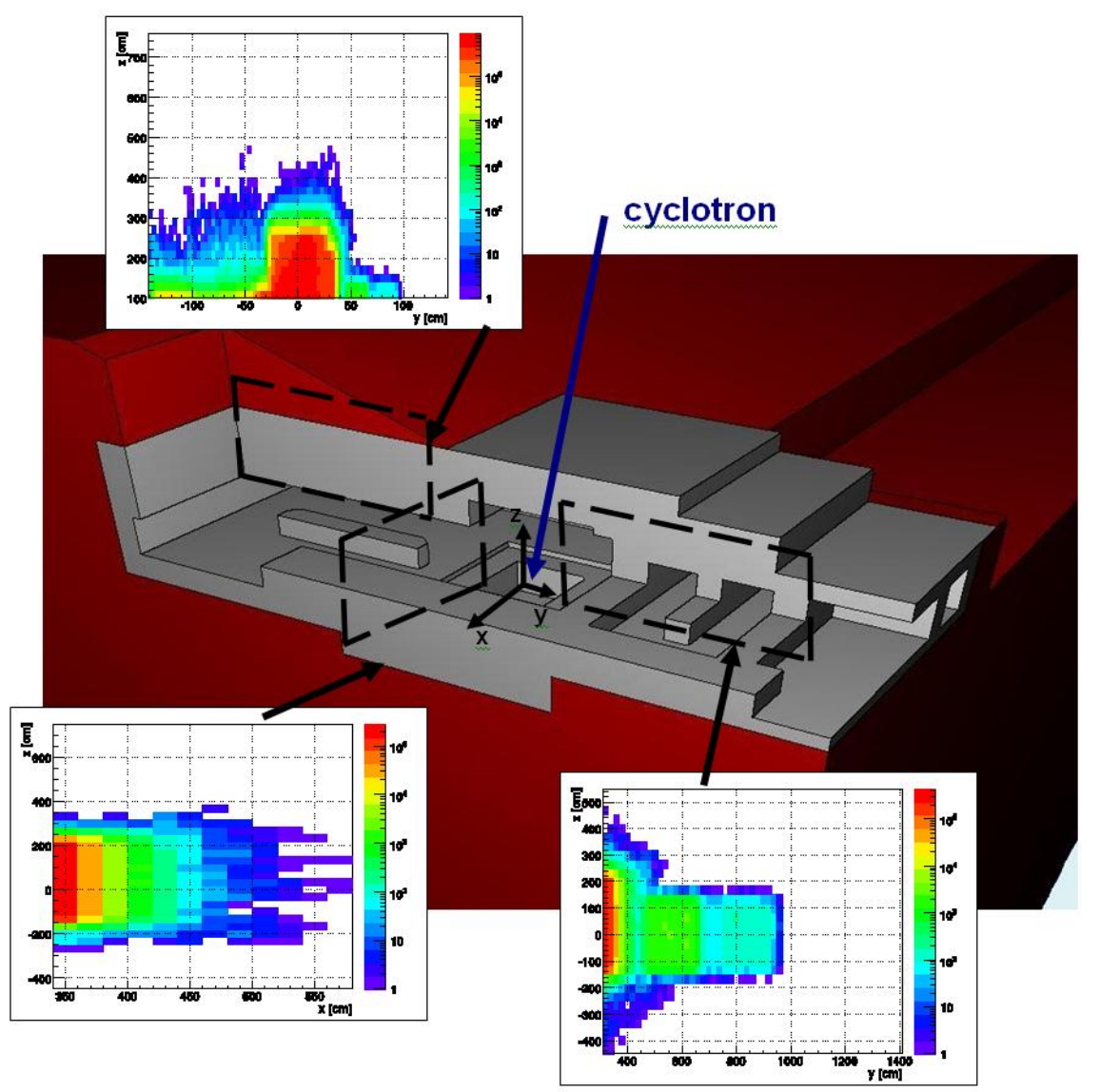

Figure 12 - (Color online) $H^{*}(10)$ [mSv/year] in selected areas of the east wall, maze and ceiling of the cyclotron room.

\section{Discussion}

The shielding barriers (concrete and eventually soil) of the cyclotron room in the Proton-Therapy-Center in Prague seem to be sufficient according to the simulation. There are also some borderline cases in a few localized areas; for example, the terraces above the entrance maze or area behind the east wall. In these critical areas there will be no permanent presence of persons, but it is recommended that they should be properly labeled. 
The uncertainty of the simulations is less than $10 \%$ (at one sigma confidence interval) in all spatial bins, in many cases even less than 5\%. These statistical uncertainties can affect the results, especially at the $1 \mathrm{mSv} / y e a r$ value outside the facility (one main objective, as indicated in the introduction). On the other hand, in the study (Newhauser et al., 2002) the comparison of experimental and calculated data is presented for different locations in the Proton Therapy Center and in almost all cases the simulations overestimate the experimental values, typically by more than a factor of 10 , and in some cases more than 100 . The simulations in (Newhauser et al., 2002) were performed with the same Monte Carlo code as in this study. Unfortunately, the experimental data from the Prague Proton-Therapy-Center are not available yet.

The area directly above the cyclotron and the energy selection system (degrader and collimator) also requires special attention. The area above the cyclotron is constructed as a removable cyclotron hatch which must be provided for lowering the accelerator into the building. That means there is no concrete plate, but only some concrete blocks. So, attention should be paid when placing these blocks to try to fit them together without large air gaps and/or to fill gaps with another shielding material. The area directly above the degrader and collimator is also a little problematic. To achieve the annual dose equivalent of $<1 \mathrm{mSv} / \mathrm{year}$ a wall of $4.20 \mathrm{~m}$ thickness of concrete is needed and the space available in this area is only $2.20 \mathrm{~m}$. To increase the ceiling it should be possible to use iron or steel as a shielding material. The NCRP Report No. 144 (NCRP, 2005) recommends that any iron or steel shielding should be backed by at least $0.61 \mathrm{~m}$ of concrete (since the cross-section for the interaction of neutrons with iron falls rapidly below about $5 \mathrm{MeV}$ ). On the other hand, and because the cyclotron room is not a place with the permanent presence of people, roof height of $2.20 \mathrm{~m}$ should be enough in some services' work.

The choice of composition of the materials in simulations can also have some influence. On the other hand, in high-energy level interactions there is much more critical value of mass density than atomic/isotope composition of material. For this reason, in this study the standard composition of shielding materials was used, but with reduced density, so that the simulated values should not be underestimated.

\section{Conclusion}

The shielding barriers of the room with the cyclotron and energy selection systems were verified. The thickness of the concrete walls proposed in the project is sufficient and meets the requirements of current legislation in the Czech Republic in all areas. Some possibly problematic areas are described above, in the Discussion section. 
Acknowledgement. This work was supported by the Grant Agency of the Czech Technical University in Prague, grant No. SGS12/200/OHK4/3T/14 and by the Ministry of Education, Youth and Sports, project No. MSM6840770040.

\section{REFERENCES}

ICRP Publication 74 (1996) Conversion coefficient for use in radiological protection against radiation, Ann. ICRP 26 (3-4).

NCRP (2005) Report No. 144, Radiation protection for particle accelerator facilities, National Council on Radiation protection and Measurements, issued December 31, 2003, revised January 7, 2005, Radiat. Prot. Dosim. 113 (4), 456-457.

Newhauser W.D. et al. (2002) Neutron shielding verification measurements and simulations for a 235$\mathrm{MeV}$ proton therapy center, Nucl. Instrum. Meth. Phys. Res. A 476, 80-84.

Pelowitz D.B. (2005) MCNPX ${ }^{\mathrm{TM}}$ user's manual, version 2.5.0.

Stichelbaut F. (2007) Radiation Sources in Praha Proton Therapy Center, December 18, 2007, presentation.

Stichelbaut F. (2008) Shielding Calculations for PT Center in Praha, Czech Republic, April 9, 2008, presentation. 